\title{
CHEMICAL HEATING IN STRANGE STARS
}

\author{
K. S. CHENG ${ }^{1}$ AND Z. G. DAI ${ }^{2}$ \\ Received 1995 October 31 ; accepted 1996 March 27
}

\begin{abstract}
As a strange star spins down, its core density increases, changing the chemical equilibrium state of strange matter. The relaxation toward the new equilibrium state takes a finite time, so the matter is not quite in chemical equilibrium, and energy is released by reactions at the expense of the stored chemical energy. We study such a chemical heating process for a spin-down strange star and find that this effect is significant at later times for weak magnetic fields. We compare the cooling of a strange star with that of an ordinary neutron star and find that the surface temperature of the strange star is much lower than that of the neutron star at the age up to $10^{8} \mathrm{yr}$. This favorable to an observational signature for strange stars.
\end{abstract}

Subject headings: dense matter - elementary particles — stars: interiors - stars: neutron

\section{INTRODUCTION}

Since it was conjectured by Witten (1984) that bulk strange quark matter may be more stable than hadronic matter in high densities, and it was shown by Farhi \& Jaffe (1984) that the existence of such quark matter is reasonable within the uncertainties inherent in a strong interaction calculation, the properties of strange stars have been studied by several authors (Alcock, Farhi \& Olinto 1986; Haensel, Zdunik, \& Shaeffer 1986; Colpi \& Miller 1992; Glendenning \& Weber 1992). Strange stars may be produced through the conversion of neutron stars via two distinctive ways: the transition from ordinary neutron stars (Olinto 1987; Dai, Wu, Lu 1995; Horvath \& Benvenuto 1988; Dai, Lu, \& Peng 1993) and that from proto-neutron stars during the collapse of supernova cores (Gentile et al. 1993; Dai, Peng, \& Lu, 1995).

Observationally, it is not easy to distinguish strange stars from neutron stars. First, for the observed mass region near $1.4 M_{\odot}$, two kinds of stellar objects have rather similar radii (Alcock et al. 1986; Haensel et al. 1986), so they have similar observational quantities such as gravitational redshifts. Second, strange stars with crusts have strong magnetic fields, and their magnetospheres should be similar to neutron stars. Thus, the radio (or X-ray or gamma-ray) radiation properties of pulsars are described equally well by strange star models and by neutron star models. Third, the glitch events in pulsars might be used to discriminate between strange and neutron stars, because the postglitch behavior is usually described by the neutron-superfluid vortex creep theory (for a review, see also Pines \& Alpar 1985). But so far it is not clear whether strange stars may explain glitches. These events are very infrequent in the sense of observation availability (the observed glitching stars number only 20). One could say that the stars with glitches (e.g., Crab and Vela) are normal neutron stars (Alpar et al. 1993), and some of the stars without glitches may be strange stars. Fourth, the minimum period $(\sim 0.5$ $\mathrm{ms}$ ) of strange stars is smaller than that of neutron stars (Madsen 1992; Dai \& Lu 1995). But one has not yet discovered a pulsar with a period less than $1 \mathrm{~ms}$.

\footnotetext{
${ }^{1}$ Department of Physics, University of Hong Kong, Hong Kong.

${ }^{2}$ Department of Astronomy, Nanjing University, Nanjing 210093, China.
}

The thermal radiation from the surface of a star is a very promising discriminant between strange stars and ordinary neutron stars. Because the neutrino energy loss rate in strange matter is much higher than that based on the modified Urca processes in neutron matter, the surface temperature of a young strange star is lower than that of an ordinary neutron star of the same age (Pizzochero 1991). In this paper we show that this conclusion is still true at the age up to $10^{8} \mathrm{yr}$ if chemical heating in spin-down strange stars is included. This could provide a definite observational signature for strange stars.

\section{MODEL}

For a strange star, its core contains $u, d$, and $s$ quarks and electrons, whose relative concentrations are adjusted by the following Urca reactions:

$$
\begin{aligned}
& u+e^{-} \rightarrow d+\bar{v}_{e}, \\
& d \rightarrow u+e^{-}+v_{e}, \\
& u+e^{-} \rightarrow s+\bar{v}_{e}, \\
& s \rightarrow u+e^{-}+v_{e} .
\end{aligned}
$$

The equilibrium concentration of particles of each kind depends on density. As the star spins down due to the magnetic dipole radiation, the centrifugal force decreases continuously, increasing the star's internal density. This changes the chemical equilibrium state throughout the core. Since the relaxation of the relative particle concentrations requires a finite amount of time, as compared with the spindown timescale, the star is never exactly in chemical equilibrium, so energy is stored. In addition, the finite departure from chemical equilibrium modifies the reaction rates (Dai et al. 1993). If the departure from equilibrium is large enough, the net effect of the reactions is to increase the thermal energy at the expense of the stored chemical energy. We refer to this heating mechanism as "chemical heating." Recently this mechanism in the neutron star models was studied by Reisenegger (1995).

To study chemical heating of a strange star, we calculate first the reaction rates and neutrino energy loss rates for reactions (1)-(4), which are not in chemical equilibrium. Following Dai et al. (1993), we calculate the Fermi integrals and obtain the act reaction rate per baryon for equations (1) 
and (2):

$$
\Gamma_{d}=6.1 \times 10^{-11} \alpha_{c} Y_{e}^{1 / 3} T_{8}^{5} \frac{42 u_{d}^{5}+420 u_{d}^{3}+714 u_{d}}{457} \mathrm{~s}^{-1}
$$

where $\alpha_{c}$ is the strong coupling constant defined by Farhi $\&$ Jaffe (1984), $\mathrm{Y}_{e}$ is the electron fraction, $T_{8}$ is the core temperature in units of $10^{8} \mathrm{~K}$, and $u_{d} \equiv \beta \delta \mu_{d} \equiv \beta\left(\mu_{u}+\mu_{e}-\mu_{d}\right)$ (here $\mu_{i}$ is the chemical potential of particles of a kind $i$ and $\beta=1 / k_{B} \mathrm{~T} ; k_{B}$ is the Boltzmann constant), and $T_{8}$ is the temperature in units of $10^{8} \mathrm{~K}$. We have assumed the baryon number density $n=0.4 \mathrm{fm}^{-3}$ for the $1.4 M_{\odot}$ strange star calculated by Alcock et al. (1986) and have used $\cos ^{2} \theta_{c}=$ 0.974 ( $\theta_{c}$ is the Cabbibo angle), which differs from that used by Dai et al. (1993). The total neutrino energy loss rate per baryon for reactions (1) and (2) is written as

$$
\begin{aligned}
\epsilon_{d}=1.7 & \times 10^{-12} \alpha_{c} Y_{e}^{1 / 3} T_{8}^{6} \\
& \times\left(1+\frac{21 u_{d}^{6}+315 u_{d}^{4}+1071 u_{d}^{2}}{457}\right) \mathrm{MeV} \mathrm{s}^{-1}
\end{aligned}
$$

When reactions (1) and (2) are in chemical equilibrium (i.e., $u_{d}=0$ ), the above equation is consistent with the result derived by Iwamoto (1982) and Duncan, Shapiro, \& Wasserman (1983), but not that the definition of the strong constant differs by a factor of 2 . by

The net rate per baryon for reactions (3) and (4) is given

$$
\Gamma_{8}=7.9 \times 10^{-12} Y_{e}^{1 / 3} T_{8}^{5} \frac{42 u_{s}^{5}+420 u_{s}^{3}+714 u_{s}}{457} \mathrm{~s}^{-1},
$$

Where $u_{s} \equiv \beta \delta \mu_{s} \equiv \beta\left(\mu_{u}+\mu_{e}-\mu_{s}\right)$. The corresponding neutrino energy loss rate per baryon is

$$
\begin{aligned}
\epsilon_{s}=2.1 & \times 10^{-13} Y_{e}^{1 / 3} T_{8}^{6} \\
& \times\left(1+\frac{21 u_{s}^{6}+315 u_{s}^{4}+1071 u_{s}^{2}}{457}\right) \mathrm{MeV} \mathrm{s}^{-1} .
\end{aligned}
$$

Although weak interactions coupling $s$ and $u$ quarks are "Cobbibo suppressed" relative to interactions coupling to $d$ and $u$ quarks in Weinberg-Salam-Glashow theory, since in reaction matrix elements $\left|p_{s} p_{u}\right| \gg\left|p_{d} p_{u}\right|$ (where $p_{i}$ is the four-dimensional momentum), the neutrino energy loss rate for reactions (3) and (4) can be of the same order of magnitude as that for reactions (1) and (2).

For reaction $u+d \leftrightarrow u+s$, its reaction rate $\left(\Gamma_{d s}\right)$ has been calculated (e.g., Wang \& Lu 1984; Madsen 1993; Heiselberg 1992), and its timescale toward an equilibrium state is estimated by

$$
\tau_{r} \sim \frac{\delta Y_{s}}{\Gamma_{d s}} \sim 0.1 T_{8}^{-2} \mathrm{~s},
$$

where $\delta Y_{s}$ is the change in the $s$-quark fraction $\left[\delta Y_{s}=\right.$ $\left.3 \delta \mu_{s} /{ }^{\xi} c\left(\pi^{2} n\right)^{1 / 3}\right]$, and we have assumed $\delta \mu_{d s} \equiv \mu_{d}-\mu_{s} \sim$ $\delta \mu_{s} \sim k_{B} T$. On the other hand, the timescale for the change $\delta Y_{s}$ resulting from spin-down due to the magnetic dipole radiation of a pulsar is approximated by

$$
\tau_{s} \sim \delta Y_{s}\left(Y_{e}^{2 / 3} \frac{\Omega \dot{\Omega}}{G \rho_{c}}\right)^{-1}>10^{8} T_{8} \mu_{30}^{-2} \sin ^{-2} \phi \Omega_{i 3}^{-2} I_{45} \mathrm{~s},
$$

where $Y_{e} \sim 10^{-3}, \Omega_{(i) 3}$ is the (initial) rotation frequency of the star in units of $10^{3} \mathrm{~s}^{-1} G$ is the gravitational constant, $\rho_{\mathrm{c}}$ is the central density, $\mu_{30}$ is the magnetic dipole moment in units of $10^{30} \mathrm{G} \mathrm{cm}^{3}, \phi$ is the angle between the rotation axis and the magnetic axis, and $I_{45}$ is the moment of inertia in units of $10^{45} \mathrm{~g} \mathrm{~cm}^{2}$. It can be seen from equations (9) and (10) that $\tau_{r} \ll \tau_{s}$ for any time, so in this paper we assume that reaction $u+d \leftrightarrow u+s$ is in chemical equilibrium. From this assumption, together with the conditions of charge neutrality and $Y_{u}+Y_{d}+Y_{s}=3$, we can find that there is only one independent variable among the particle concentrations $\left(Y_{u}, Y_{d}, Y_{s}\right.$, and $\left.Y_{e}\right)$. We choose $Y_{s}$, which, for simplicity, is denoted as $x$ in the rest of this paper.

Following Reisenegger (1995), we write the time evolution of the chemical-potential difference $\left(\varepsilon \mu \equiv \delta \mu_{d}=\delta \mu_{s}\right)$

$$
\frac{d \delta \mu}{d t}=-E_{x x}\left(\frac{n E_{n x}}{E_{x x}} \frac{\Omega \dot{\Omega}}{G \rho_{c}}-\Gamma_{d}-\Gamma_{s}\right),
$$

where $E_{n x}$ and $E_{x x}$ are partial derivatives of the chemical energy per baryon of strange matter, and they will be evaluated later. In this expression, the first term in parentheses accounts for the change in the chemical equilibrium due to spin-down, and the second and third terms account for the change in the actual chemical state due to the reactions. The thermal evolution is determined by

$$
C_{v} \frac{d T}{d t}=\left(\Gamma_{d}+\Gamma_{s}\right) \delta \mu-\left(\epsilon_{d}+\epsilon_{s}\right)-\dot{E}_{\gamma}
$$

where the three terms represent the chemical energy released by the reactions, the energy radiated by neutrinos and antineutrinos, and the energy in photons from the surface of the star, respectively. $C_{v}$ is the specific heat of strange matter (Iwamoto 1982),

$$
\begin{aligned}
C_{v}= & \pi^{4 / 3} n^{-1 / 3}\left(\bar{\xi}^{\bar{c}} c\right)^{-1} k_{B}^{2} T\left(Y_{u}^{2 / 3}+Y_{d}^{2 / 3}\right. \\
& \left.+T_{s}^{2 / 3}+3^{-1 / 3} Y_{e}^{2 / 3}\right)_{\mathrm{eq}} .
\end{aligned}
$$

We have assumed that the departure from the equilibrium state is small so that $Y_{i}$ values are approximately equal to their the equilibrium values referred to by the subscript "eq." For $\dot{E}_{\gamma}$, we adopt the relation derived by Gudmundsson, Pathick, \& Epstein (1983) and obtain $\dot{E}_{\gamma}=$ $2.8 \times 10^{-19} T_{8}^{2.2} \mathrm{MeV} \mathrm{s}^{-1}$.

In order to estimate $E_{n x}$ and $E_{x x}$, we assume that strange matter is an ideal Fermi-Dirac gas. So we have

$$
\frac{n E_{n x}}{E_{x x}} \approx\left[\frac{Y_{d}^{2 / 3} Y_{s}^{-1 / 3}-\left(3^{1 / 3} Y_{e}^{1 / 3}+Y_{u}^{1 / 3}\right)\left(1+Y_{d}^{1 / 3} Y_{s}^{-1 / 3}\right)}{3^{1 / 3} Y_{e}^{-2 / 3}\left(1+Y_{d}^{1 / 3} Y_{s}^{-1 / 3}\right)^{2}}\right]_{\mathrm{eq}} .
$$

and

$$
E_{x x} \approx 3^{-2 / 3} \pi^{2 / 3}\left({ }^{\bar{\xi}} c\right) n^{1 / 3}\left[Y_{e}^{2 / 3}\left(1+Y_{d}^{1 / 3} Y_{s}^{-1 / 3}\right)^{2}\right]_{\mathrm{eq}} .
$$

\section{RESULTS AND DISCUSSION}

In actual calculations, we take $n=0.4 \mathrm{fm}^{-3}$, which is the typical value for the $1.4 M_{\odot}$ strange star calculated by Alcock et al. (1986). The equilibrium state particle concentrations are found by using the thermodynamic potentials 
expressed by Farhi \& Jaffe (1984) when the strong coupling constant and S-quark mass are taken into account. Thus, $C_{v}, n E_{n x} / E_{x x}$, and $E_{x x}$ are calculated. Assuming that spindown of the star is due to the magnetic dipole radiation, we have

$$
-\frac{\Omega \dot{\Omega}}{G \rho_{c}} \approx \frac{0.01 \Omega_{i 3}^{2}}{\tau(1+t / \tau)^{2}},
$$

where $\tau=2.1 \times 10^{10} \mu_{30}^{-2} \sin ^{-2} \phi \Omega_{i 3}^{-2} I_{45} \quad \mathrm{~s}$ with an assumed moment of inertia $i \equiv 10^{45} \mathrm{~g} \mathrm{~cm}^{2}$.

We assume an initial rotation period $P_{i}=1 \mathrm{~ms}$, but this value does not affect the cooling curve sensitively. After giving the surface magnetic field strength, the strong coupling constant, and the s-quark mass, we integrate the coupling evolution equations (11) and (12). Figure 1 shows the evolution of the effective surface temperature for $B=10^{12}$ $\mathrm{G}$ (dotted line), $10^{11} \mathrm{G}$ (dot-dashed line), $10^{10} \mathrm{G}$ (shortdashed line), and $10^{9} \mathrm{G}$ (long-dashed line), and in the absence of chemical heating (solid line). In this figure, $\alpha_{c}$ is taken to be 0.1 , and $m_{s}$ is taken to be $200 \mathrm{MeV}$. One sees that the effect of chemical heating can be substantial at later times for stars with weak fields but be quite weak for stars with strong fields.

As a comparison, Figure 2 gives the evolution of the effective surface temperature for strange stars with $\alpha_{c}=0$ and $m_{s}=300 \mathrm{MeV}$ (bottom curves) and ordinary neutron stars with modified Urca processes (top curves). Our results for neutron stars are consistent with those given by Reisenegger (1995). It is obvious that the effect of chemical heating is to increase the difference between the surface temperatures of strange stars and of neutron stars. This result means that even at later times of a pulsar, one could also decide whether it is a strange star or an ordinary neutron star based on the observed surface temperature.

Although the recent $R O S A T$ observations (Ögelman 1995) did not find any evidence for the existence of cold young pulsars that are expected to be strange stars, we would like to point out that there may still exist young strange stars. However, these young stars are just not many, and they are too cold and too far away to be observed. On the other hand, old and nearby strange stars could be as numerous as old neutron stars, and their magnetic field can be as weak as $10^{9} \mathrm{G}$ (Chau 1996). From Figure 2, we can

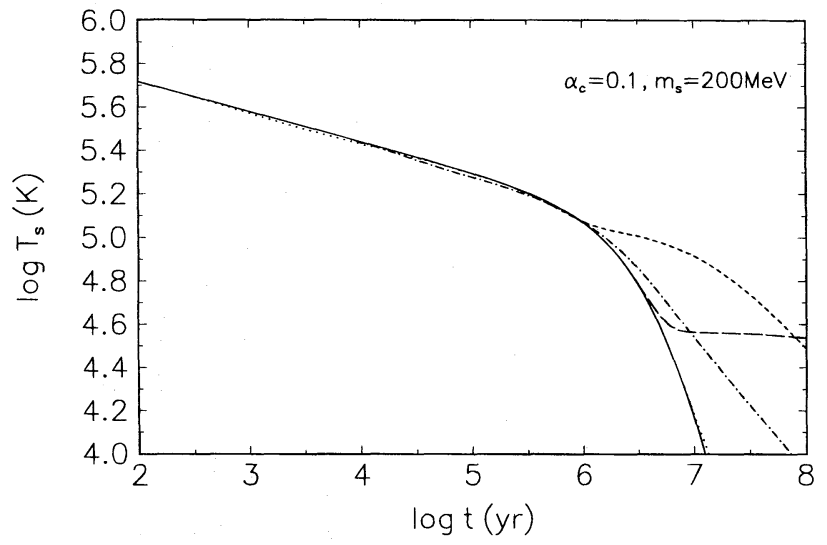

FIG. 1.-Effective surface temperature as a function of time for strange stars with $\alpha_{c}=0.1$ and $m_{s}=100 \mathrm{MeV}$ for no heating (solid line) or spindown heating with magnetic field strengths $B=10^{12} \mathrm{G}$ (dotted line), $10^{11}$ $\mathrm{G}$ (dot-dashed line), $10^{10} \mathrm{G}$ (short-dashed line), and $10^{9} \mathrm{G}$ (long-dashed line). The initial rotation period is taken to be $1 \mathrm{~ms}$.

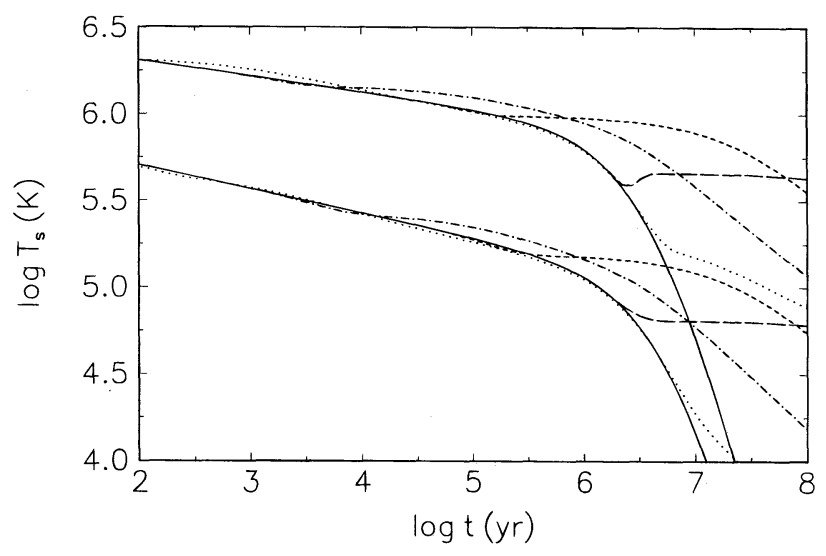

FIG. 2.-Effective surface temperature as a function of time for strange stars with $\alpha_{c}=0$ and $m_{s}=300 \mathrm{MeV}$ (bottom lines) and standard neutron stars (top lines). Curves are shown as in Fig. 1.

distinguish easily between a weak field neutron star and a strange star. The latter will be much hotter than from the former.

In Figure 3 we show that, as $m_{s}$ increases (or $\alpha_{c}$ decreases) for strange stars, the effect of chemical heating becomes more and more significant at latter times. Thus, once a star is confined to be a strange star, we may determine the values of $m_{s}$ and $\alpha_{c}$ by observing the surface temperature.

We have considered the case of cooling of neutron stars with modified Urca processes. In the interiors of neutron stars, however, direct Urca processes can proceed when the proton concentration of matter exceeds the threshold value (=11.1\%), as discussed by Lattimer et al. (1991). This possibility has been realized recently by Sumiyoshi, Kuwabara, \& Toki 1995, where neutron matter is described by the relativistic mean-field theory with the two sets of parameters denoted as NL1 and TM1. Besides the state with high proton concentration, neutron-star matter may be in another exotic state, such as a pion condensate or a kaon condensate (for a recent review, see Pethick 1992). These states could lead to copious neutrino emission and rapid cooling of neutron stars. We plan to study chemical heating of spin-down neutron stars with these exotic states and compare it with that of strange stars elsewhere.

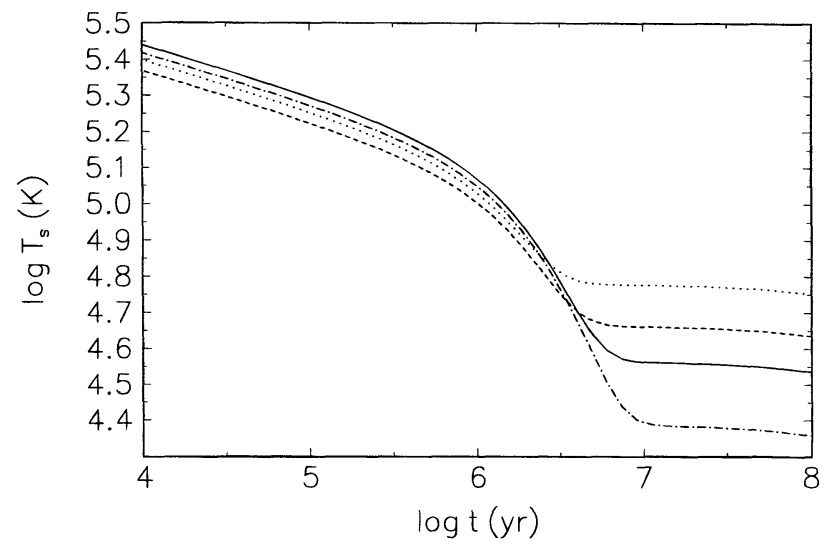

FIG. 3.-Effective surface temperature as a function of time for strange stars with $B=10^{9} \mathrm{G}$ for $\alpha_{c}=0.1$ and $m_{s}=200 \mathrm{MeV}$ (solid line), $\alpha_{c}=0.1$ and $m_{s}=300 \mathrm{MeV}$ (dotted line), $\alpha_{c}=0.4$ and $m_{s}=200 \mathrm{MeV}$ (dot-dashed line), and $\alpha_{c}=0.4$ and $m_{s}=300 \mathrm{MeV}$ (dashed line). 
Alcock, C., Farhi, E., \& Olinto, A. 1986, ApJ, 310, 261

Alpar, M. A., Chau, H. F., Cheng, K. S., \& Pines, D. 1993, ApJ, 409, 345

I: Chau, H. F. 1996, Princeton preprint

$\therefore$ Colpi, M., \& Miller, J. C. 1992, ApJ, 388, 513

Dai, Z. G., \& Lu, T. 1995, Phys. Rev. D, submitted

Dai, Z. G., Lu, T., \& Peng, Q. H. 1993, Phys. Lett., B319, 199

In' Dai, Z. G., Peng, Q. H., \& Lu, T. 1995, ApJ, 440, 815

Dai, Z. G., Wu, X. J., \& Lu, T. 1996, Ap\&SS, in press

- I Duncan, R. C., Shapiro, S. L., \& Wasserman, I. 1983, ApJ, 267, 358

Farhi, E., \& Jaffe, R. L. 1984, Phys. Rev., D30, 3279

Gentile, N. A., Aufderheide, M. B., Mathews, G. J., Swesty, F. D., \& Fuller, G. M. 1993, ApJ, 414, 701

Glendenning, N. K., \& Weber, F 1992, ApJ, 400, 647

Gudmundsson, E. H., Pethick, J. C. \& Epstein, R. I. 1983, ApJ, 272, 286

Haensel, P., Zdunik, J. L., \& Shaeffer, R. 1986, A\&A, 160, 121

Heiselberg, H. 1992, Phys. Scr., 46, 485

\section{REFERENCES}

Horvath, J. E., \& Benvenuto, O. G. 1988, Phys. Lett., B213, 516

Iwamoto, N. 1982, Ann. Phys., 141, 1

Lattimer, J. M., Pethick, C. J., Prakash, M., \& Haensel, P. 1991, Phys. Rev. Lett., 66, 2701

Madsen, J. 1992, Phys. Rev., D46, 3290

. 1993, Phys. Rev., D47, 325

Ögelman, H. B. 1995, in The Lives of the Neutron Stars, ed. M. A. Alpar, U. Kilziloglu, \& J. van Paradijs (Dordrecht: Kluwer), 101

Olinto, A. 1987 Phys. Lett., B192, 71

Pethick, C. J. 1992, Rev. Mod. Phys., 64, 1133

Pines, D., \& Alpar, M. A. 1985, Nature, 316, 27

Pizzochero, P. 1991, Phys. Rev. Lett., 66, 2425

Reisenegger, A. 1995, ApJ, 442, 749

Sumiyoshi, K., Kuwabara, H., \& Toki, H. 1995, Nucl. Phys., A581, 725

Wang, Q. D., \& Lu, T. 1984, Phys. Lett., B148, 211

Witten, E. 1984, Phys. Rev., D30, 272 